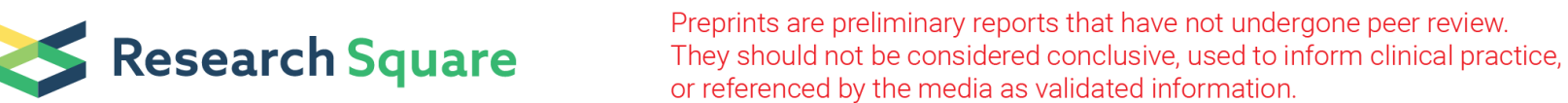

\section{Availability and use of therapeutic interchange policies in managing antimicrobial shortages among South African public sector hospitals}

\section{Audrey Komborerai Chigome}

Sefako Makgatho Health Sciences University

\section{Moliehi Matlala}

Sefako Makgatho Health Sciences University

\section{Brian Godman}

University of Strathclyde

Johanna Catharina Meyer ( $\sim$ hannelie.meyer@smu.ac.za )

Sefako Makgatho Health Sciences University https://orcid.org/0000-0003-0462-5713

Research article

Keywords: antimicrobial shortages, antimicrobial stewardship, therapeutic interchange, pharmacists, public sector hospitals, South Africa

Posted Date: August 28th, 2019

DOI: https://doi.org/10.21203/rs.2.10968/v1

License: (c) (i) This work is licensed under a Creative Commons Attribution 4.0 International License. Read Full License 


\section{Abstract}

Background: Therapeutic interchange policies in hospitals are useful in dealing with antimicrobial shortages and minimising resistance rates. However, the extent of antimicrobial shortages and availability of therapeutic interchange policies is unknown among public sector hospitals in South Africa. Objective This study aimed to ascertain the extent of antimicrobial shortages among public sector hospitals, the presence of current therapeutic interchange policies and the role of pharmacists in the process. Setting Public sector hospitals in South Africa. Methods: A quantitative and descriptive study was conducted with a target population of 403 public sector hospitals. Data were collected from hospital pharmacists using an electronic questionnaire, administered via SurveyMonkeyTM. Main outcome measure Prevalence of public sector hospitals with antimicrobial shortages over the past six months and the prevalence of hospitals with therapeutic interchange policies. Results: The response rate was $33.5 \%$. Most (83.3\%) hospitals had experienced shortages in the previous six months. Antimicrobials commonly reported as out of stock included cloxacillin (54.3\%), benzathine benzylpenicillin (54.2\%), erythromycin (39.6\%) and ceftriaxone (38.0\%). Reasons for shortages included pharmaceutical companies with supply constraints $(85.3 \%)$ and an inefficient supply system. Only $42.4 \%$ had therapeutic interchange policies, and $88.9 \%$ contacted the prescriber when there was a need for substitution. Conclusions: Antimicrobial shortages are prevalent in South African public sector hospitals with penicillins and cephalosporins being the most affected. Therapeutic interchange policies are not available at most hospitals. Effective strategies are required to improve communication between pharmacists and prescribers to ensure safe, appropriate and therapeutically equivalent alternatives are available.

\section{Background}

Sub-Saharan countries, including South Africa, have the highest burden of infectious diseases worldwide, enhanced by high prevalence rates of HIV, malaria and tuberculosis [1-3], with antimicrobials crucial to reduce the burden of communicable diseases [4]. On one hand, there are concerns with inappropriate use of antibiotics leading to increasing rates of antimicrobial resistance (AMR) limiting therapeutic options, which have resulted in antimicrobial stewardship programmes (ASPs) across countries acknowledging the challenges [5-8]. However, shortages of antimicrobials are also a challenge in Africa [9-11], potentially resulting in the use of antibiotics that place patients at greater risk of Clostridium difficile infections as well as increased AMR $[12,13]$.

The medicine shortage situation in South Africa is also a concern receiving media attention [14-16]. As a result, national monitoring programmes have been put into place in South Africa with the support of the National Department of Health ( $\mathrm{NDoH}$ ) surrounding improved supplies management as well as the 'Stop Stockouts' initiative $[17,18]$. Despite these initiatives, shortages are still being experienced in some provinces in South Africa $[16,18,19]$.Shortages of antimicrobials are regarded as a public health emergency due to the necessity to expedite treatment in the case of an infection and because escalating AMR rates limit therapeutic options for many pathogens [20,21]. 
Where shortages of antimicrobials exist, therapeutic interchange policies can potentially help ensure that substitution is not haphazard or inappropriate [22]. Pharmacists can play a crucial role in the development and implementation of therapeutic interchange policies in hospitals combined with other key stakeholder groups, building on activities within Pharmacy and Therapeutic Committees (PTCs) as well as ASPs $[8,23,24]$. Whilst the effective functioning of PTCs is improving in South Africa and ASPs are evolving $[23,24]$, there are concerns regarding the extent of therapeutic interchange policies among public hospitals in South Africa especially regarding antimicrobials. The NDoH published a policy in 2017 to provide guidance for the placement of medicines in classes to support therapeutic interchange programmes where pertinent [25]. However, the policy only provides guidelines on required procedures when switching a patient from one medicine to another within the same therapeutic class, but not on therapeutic interchanges where this is not possible such as antimicrobials. As a result, there is a need to build on this initiative.

Consequently, this study sought to identify and describe current therapeutic interchange policies in the event of drug shortages among public sector hospitals in South Africa following the NDoH initiative. Antimicrobials were chosen in view of the extent of infectious diseases in sub-Saharan Africa, with public sector hospitals chosen as they offer health services to approximately $80 \%$ of the population in South Africa [17]. The findings can be used to guide future policies in South Africa.

\section{Materials And Methods}

We used a descriptive survey design and a quantitative research approach. All public sector hospitals including district, regional and tertiary/academic hospitals $(n=403)$ in the nine provinces of South Africa were targeted for participation. One pharmacist from each hospital, in most instances the 'Drug Controller', 'Procurement Pharmacist', or 'Pharmacy Manager', were purposively selected to complete the questionnaire. In hospitals where none of these were available, a qualified pharmacist or community service pharmacist was requested to participate.

Data were collected from March to July 2018. The survey was conducted using a 23 item electronic questionnaire administered via SurveyMonkey ${ }^{\mathrm{T}}$. A questionnaire link was sent via email or fax to pharmacists who agreed to take part with anonymous completion. The questionnaire was based on the published literature [11, 22, 26-29] and followed by expert review. It was divided into three sections: demographics, antimicrobial shortages and therapeutic interchange. The questionnaire also included information on current PTC and ASP activities in the hospitals.

Data were exported from SurveyMonkey ${ }^{\mathrm{TM}}$ to Microsoft Office Excel ${ }^{\mathrm{TM}}$ and cleaned prior to analysis using the Statistical Package for the Social Sciences (SPSS) version 25. Open-ended responses were typed into MS Excel ${ }^{T M}$, and relevant categories were created to allow for counting of responses. Descriptive statistics were used to summarise data using means with standard deviation (SD) and frequency counts with percentages. Antibiotics were classified by ATC class [30]. All analyses were conducted at a $95 \%$ confidence interval. 
Ethical clearance for the study was granted by the Sefako Makgatho University Research Ethics Committee (SMUREC/P/269/2017:PG), and permission obtained from the NDoH.

\section{Results}

\section{Response rate and demographic details}

Of the 403 hospitals, approval to conduct the study was received for 346 hospitals (Table 1). Of these 346 hospitals, 81 facilities could not be reached using email addresses and 11 declined to participate. A total of 85 responses were finally received, giving a response rate of 33.5\% (Table 1).

Table 1 Response rate and study population per province

\begin{tabular}{|c|c|c|c|c|c|c|c|c|}
\hline \multirow[t]{2}{*}{ Province } & \multirow[b]{2}{*}{$\begin{array}{l}\text { Target } \\
\text { popu- } \\
\text { lation } \\
(\mathrm{n}=403)\end{array}$} & \multicolumn{4}{|c|}{ Excluded from target population $(\mathrm{n}=149)$} & \multicolumn{3}{|c|}{ Study population $(n=254)$} \\
\hline & & $\begin{array}{l}\text { No } \\
\text { approval } \\
\text { response }\end{array}$ & $\begin{array}{l}\text { Approval } \\
\text { denied }\end{array}$ & $\begin{array}{l}\text { Could not } \\
\text { be reached } \\
\text { for an email } \\
\text { address }\end{array}$ & $\begin{array}{l}\text { Declined } \\
\text { participation/ no } \\
\text { pharmacist/ no } \\
\text { email or fax }\end{array}$ & $\begin{array}{l}\text { Email } \\
\text { delivered }\end{array}$ & $\begin{array}{l}\text { Non- } \\
\text { response }\end{array}$ & $\begin{array}{l}\text { Responses } \\
\text { per } \\
\text { province } \\
(\%)\end{array}$ \\
\hline $\begin{array}{l}\text { Eastern } \\
\text { Cape }\end{array}$ & 90 & 0 & $\overline{0}$ & 28 & 3 & 59 & 41 & $\begin{array}{l}18 \\
(21.2 \%)\end{array}$ \\
\hline Free State & 34 & 0 & 1 & 17 & 2 & 14 & 8 & $6(7.1 \%)$ \\
\hline Gauteng & 36 & 26 & 0 & 0 & 0 & 10 & 2 & $8(9.4 \%)$ \\
\hline $\begin{array}{l}\text { Kwa-Zulu } \\
\text { Natal }\end{array}$ & 77 & 0 & 0 & 7 & 0 & 70 & 57 & $\begin{array}{l}13 \\
(15.3 \%)\end{array}$ \\
\hline Limpopo & 40 & 0 & 0 & 4 & 0 & 36 & 28 & $8(9.4 \%)$ \\
\hline Mpumalanga & 33 & 0 & 0 & 11 & 1 & 21 & 17 & $4(4.7 \%)$ \\
\hline $\begin{array}{l}\text { Northern } \\
\text { Cape }\end{array}$ & 19 & 0 & 0 & 1 & 3 & 15 & 2 & $\begin{array}{l}13 \\
(15.3 \%)\end{array}$ \\
\hline North West & 20 & 0 & 0 & 1 & 1 & 18 & 10 & $8(9.4 \%)$ \\
\hline $\begin{array}{l}\text { Western } \\
\text { Cape }\end{array}$ & 54 & 20 & 10 & 12 & 1 & 11 & 4 & $7(8.2 \%)$ \\
\hline $\begin{array}{l}\text { Total } \\
\text { number (\%) }\end{array}$ & 403 & $\begin{array}{l}46 \\
(11.4 \%) \\
\end{array}$ & $\begin{array}{l}11 \\
(2.7 \%) \\
\end{array}$ & $81(20.1 \%)$ & $11(2.7 \%)$ & 254 & $\begin{array}{l}169 \\
(66.5 \%) \\
\end{array}$ & $\begin{array}{l}85 \\
(33.5 \%) \\
\end{array}$ \\
\hline
\end{tabular}

More than half of the respondents were female (63.5\%), with a mean age of 38 years (SD: 9.8) (Table 2). The mean number of years in practice was 9.3 years (SD: 7.42), with 62.2\% having less than 10 years experience. Almost half (48.2\%) were pharmacy managers, with most working at district hospitals (Table 2). 
Table 2 Demographic characteristics of respondents $(n=85)$

\begin{tabular}{|c|c|c|}
\hline Respondents' characteristics & & $\begin{array}{l}\text { Respondents; } \mathrm{n} \\
(\%)\end{array}$ \\
\hline \multirow[t]{2}{*}{ Gender } & Male & $31(36.5 \%)$ \\
\hline & Female & $54(63.5 \%)$ \\
\hline \multirow[t]{4}{*}{ Age (years) } & $20-30$ & $19(24.1 \%)$ \\
\hline & प30-40 & $28(35.4 \%)$ \\
\hline & $\square 40-50$ & $20(25.3 \%)$ \\
\hline & प50-65 & $12(15.2 \%)$ \\
\hline \multirow{4}{*}{$\begin{array}{l}\text { Years of practice in the public } \\
\text { sector }\end{array}$} & $\leq 10$ & $51(62.2 \%)$ \\
\hline & प10-20 & $24(29.3 \%)$ \\
\hline & प20-30 & $5(6.1 \%)$ \\
\hline & $\square 30$ & $2(2.4 \%)$ \\
\hline \multirow[t]{9}{*}{ Designation } & Pharmacy manager & $41(48.2 \%)$ \\
\hline & $\begin{array}{l}\text { Pharmacist (completed community service; no specific } \\
\text { designation) }\end{array}$ & $19(22.4 \%)$ \\
\hline & Procurement Pharmacist & $13(15.3 \%)$ \\
\hline & Drug controller & $10(11.8 \%)$ \\
\hline & Clinical Pharmacist & $4(4.7 \%)$ \\
\hline & Community Service Pharmacist & $4(4.7 \%)$ \\
\hline & Production Pharmacist & $2(2.4 \%)$ \\
\hline & Clinical supervisor & $1(1.2 \%)$ \\
\hline & Sub-district Pharmacist & $1(1.2 \%)$ \\
\hline \multirow[t]{5}{*}{ Level of care } & District hospital & $43(51.8 \%)$ \\
\hline & Regional hospital & $17(20.5 \%)$ \\
\hline & Tertiary hospital & $11(13.3 \%)$ \\
\hline & Specialised hospital & $10(12.1 \%)$ \\
\hline & Central hospital & $3(3.6 \%)$ \\
\hline
\end{tabular}

\section{Antimicrobial shortages}

The majority of respondents (83.3\%) indicated they had experienced antimicrobial shortages at their institutions in the previous six months. Most shortages were for antibiotics particularly penicillins, with a limited number in the antiviral (acyclovir) and antifungal (amphoteracin B, clotrimazole) classes (Table 3). Most of the shortages exceeded 40 days.

Table 3 Antimicrobial shortages by ATC classification 


\begin{tabular}{|c|c|c|c|c|c|}
\hline Antimicrobial class & $\begin{array}{l}\text { ATC } \\
\text { classification }\end{array}$ & Antimicrobial & $\begin{array}{l}\text { Number of } \\
\text { respondents }^{a}\end{array}$ & $\begin{array}{l}\text { Total number (\%) } \\
\text { who reported } \\
\text { antimicrobial as } \\
\text { unavailable }\end{array}$ & $\begin{array}{l}\text { Average } \\
\text { duration of } \\
\text { shortages } \\
\text { (days) }\end{array}$ \\
\hline \multirow[t]{7}{*}{ Penicillins } & J01CF02 & Cloxacillin & 70 & $38(54.3 \%)$ & 5 to $>40$ \\
\hline & J01CE08 & $\begin{array}{l}\text { Benzathine } \\
\text { Benzylpenicillin }\end{array}$ & 72 & $39(54.2 \%)$ & 5 to $>40$ \\
\hline & J01CE02 & $\begin{array}{l}\text { Phenoxymethylpenicillin } \\
\text { IV }\end{array}$ & 58 & $28(48.3 \%)$ & 21 to $>40$ \\
\hline & J01CE02 & $\begin{array}{l}\text { Phenoxymethylpenicillin } \\
\text { oral }\end{array}$ & 71 & $31(43.7 \%)$ & 5 to $>40$ \\
\hline & J01CE01 & Benzyl Penicillin & 72 & $24(33.3 \%)$ & 5 to $>40$ \\
\hline & J01CE09 & Procaine Penicillin & 55 & $14(25.5 \%)$ & 21 to $>40$ \\
\hline & J01CA01 & Ampicillin IV & 72 & 15 (20.8\%) & 5 to $>40$ \\
\hline \multirow[t]{2}{*}{$\begin{array}{l}\text { B-lactam inhibitor } \\
\text { combinations }\end{array}$} & J01CR02 & $\begin{array}{l}\text { Amoxicillin/Clavulanic } \\
\text { Acid IV }\end{array}$ & 71 & $21(29.6 \%)$ & 5 to $>40$ \\
\hline & J01CR05 & Piperacillin/Tazobactam & 61 & $16(26.2 \%)$ & 5 to 40 \\
\hline \multirow[t]{2}{*}{ Macrolides } & J01FA01 & Erythromycin & 53 & $21(39.6 \%)$ & 5 to $>40$ \\
\hline & J01FA10 & Azithromycin IV & 59 & $11(18.6 \%)$ & 11 to 40 \\
\hline \multirow[t]{2}{*}{ Cephalosporins } & J01DD04 & Ceftriaxone & 71 & $27(38.0 \%)$ & 5 to $>40$ \\
\hline & J01DE01 & Cefepime & 53 & $14(26.4 \%)$ & 5 to $>40$ \\
\hline Aminoglycosides & J01GB03 & Gentamicin & 67 & $19(28.4 \%)$ & 5 to $>40$ \\
\hline $\begin{array}{l}\text { Imidazole } \\
\text { antifungal }\end{array}$ & D01AC01 & Clotrimazole cream & 71 & $20(28.2 \%)$ & 5 to $>40$ \\
\hline \multirow[t]{2}{*}{ Glycopeptides } & J01XA01 & Vancomycin Oral & 40 & $11(27.5 \%)$ & $>40$ \\
\hline & J01XA01 & Vancomycin IV & 62 & $13(21.0 \%)$ & 11 to 40 \\
\hline $\begin{array}{l}\text { Synthetic } \\
\text { nucleoside } \\
\text { analogue antiviral }\end{array}$ & D06BB03 & Aciclovir IV & 58 & $14(24.1 \%)$ & 5 to $>40$ \\
\hline $\begin{array}{l}\text { Tetraene polyene } \\
\text { antifungal }\end{array}$ & D01AA02 & Natamycin & 43 & $8(18.6 \%)$ & $>40$ \\
\hline Polyene antifungal & J02AA01 & Amphoteracin B & 72 & $13(18.1 \%)$ & 5 to $>40$ \\
\hline
\end{tabular}

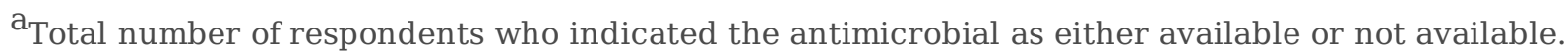

\section{Reasons for antimicrobial shortages}

The majority of respondents (85.3\%) stated that supply problems with pharmaceutical companies were the main contributor to shortages (Table 4). The impact included dispensing later generation (84.4\%) and more expensive alternatives (64.9\%). The procedures for reporting medicine shortages included sending weekly reports to the central/ provincial office or district pharmacist (Table 4).

Table 4 Reasons for, impact of, and procedures for reporting antimicrobial shortages 


\begin{tabular}{|c|c|c|c|c|c|c|}
\hline \multirow[b]{2}{*}{ Reasons for antimicrobial shortages ${ }^{a}$} & \multicolumn{5}{|c|}{ Hospital level of care } & \multirow[b]{2}{*}{$\begin{array}{l}\text { Total } \\
(\%) \\
(\mathrm{n}=75) \\
\end{array}$} \\
\hline & $\begin{array}{l}\text { District } \\
(\mathrm{n}=37)\end{array}$ & $\begin{array}{l}\text { Regional } \\
(\mathrm{n}=15)\end{array}$ & $\begin{array}{l}\text { Tertiary } \\
(\mathrm{n}=10)\end{array}$ & $\begin{array}{l}\text { Central } \\
(\mathrm{n}=3)\end{array}$ & $\begin{array}{l}\text { Specialised } \\
(\mathrm{n}=10)\end{array}$ & \\
\hline $\begin{array}{l}\text { Pharmaceutical companies with supply or } \\
\text { capacity problems }\end{array}$ & $\begin{array}{l}33 \\
(51.6 \%)\end{array}$ & $\begin{array}{l}11 \\
(17.2 \%)\end{array}$ & $\begin{array}{l}7 \\
(10.9 \%)\end{array}$ & $\begin{array}{l}3 \\
(4.7 \%)\end{array}$ & $10(15.6 \%)$ & $\begin{array}{l}64 \\
(85.3 \%)\end{array}$ \\
\hline $\begin{array}{l}\text { Inefficient supply system from the depot to the } \\
\text { facility }\end{array}$ & $\begin{array}{l}31 \\
(56.4 \%)\end{array}$ & $\begin{array}{l}10 \\
(11.1 \%)\end{array}$ & $\begin{array}{l}8 \\
(14.5 \%)\end{array}$ & $\begin{array}{l}1 \\
(1.8 \%)\end{array}$ & $5(9.1 \%)$ & $\begin{array}{l}55 \\
(73.3 \%)\end{array}$ \\
\hline Poor stock control systems & $\begin{array}{l}9 \\
(50.0 \%)\end{array}$ & $\begin{array}{l}5 \\
(27.8 \%)\end{array}$ & $\begin{array}{l}2 \\
(11.1 \%)\end{array}$ & $\begin{array}{l}0 \\
(0.0 \%)\end{array}$ & $2(11.1 \%)$ & $\begin{array}{l}18 \\
(24.0 \%)\end{array}$ \\
\hline Shortage of funds/ resources & $\begin{array}{l}9 \\
(50.0 \%)\end{array}$ & $\begin{array}{l}5 \\
(27.8 \%)\end{array}$ & $\begin{array}{l}2 \\
(11.1 \%)\end{array}$ & $\begin{array}{l}1 \\
(5.6 \%)\end{array}$ & $1(5.6 \%)$ & $\begin{array}{l}18 \\
(24.0 \%)\end{array}$ \\
\hline Wastage of medicines & $\begin{array}{l}5 \\
(35.7 \%)\end{array}$ & $\begin{array}{l}3 \\
(21.4 \%)\end{array}$ & $\begin{array}{l}3 \\
(21.4 \%)\end{array}$ & $\begin{array}{l}1 \\
(7.1 \%)\end{array}$ & $2(14.3 \%)$ & $\begin{array}{l}14 \\
(18.7 \%)\end{array}$ \\
\hline $\begin{array}{l}\text { Increase in the number of patients relying on the } \\
\text { facility for medication }\end{array}$ & $\begin{array}{l}7 \\
(50.0 \%)\end{array}$ & $\begin{array}{l}4 \\
(28.6 \%)\end{array}$ & $\begin{array}{l}2 \\
(14.3 \%)\end{array}$ & $\begin{array}{l}0 \\
(0.0 \%)\end{array}$ & $1(7.1 \%)$ & $\begin{array}{l}14 \\
(18.7 \%)\end{array}$ \\
\hline $\begin{array}{l}\text { Lack of reliable information on medicine needs } \\
\text { and usage }\end{array}$ & $\begin{array}{l}4 \\
(33.3 \%)\end{array}$ & $\begin{array}{l}4 \\
(33.3 \%)\end{array}$ & $\begin{array}{l}4 \\
(33.3 \%)\end{array}$ & $\begin{array}{l}0 \\
(0.0 \%)\end{array}$ & $0(0.0 \%)$ & $\begin{array}{l}12 \\
(16.0 \%)\end{array}$ \\
\hline Unclear lines of accountability & $\begin{array}{l}5 \\
(71.4 \%)\end{array}$ & $0(0.0 \%)$ & $\begin{array}{l}1 \\
(14.3 \%)\end{array}$ & $\begin{array}{l}0 \\
(0.0 \%)\end{array}$ & $1(14.3 \%)$ & $\begin{array}{l}7 \\
(9.3 \%)\end{array}$ \\
\hline Poor ordering practices by pharmacists or nurses & $\begin{array}{l}4 \\
(80.0 \%)\end{array}$ & $0(0.0 \%)$ & $\begin{array}{l}1 \\
(20.0 \%)\end{array}$ & $\begin{array}{l}0 \\
(0.0 \%)\end{array}$ & $0(0.0 \%)$ & 5 \\
\hline Protest action resulting in shut down of depot & $\begin{array}{l}4 \\
(80.0 \%)\end{array}$ & $\begin{array}{l}1 \\
(20.0 \%)\end{array}$ & $\begin{array}{l}0 \\
(0.0 \%)\end{array}$ & $\begin{array}{l}0 \\
(0.0 \%)\end{array}$ & $0(0.0 \%)$ & $\begin{array}{l}5 \\
(6.7 \%)\end{array}$ \\
\hline Lack of storage facilities for medicines & $\begin{array}{l}3 \\
(100.0 \%)\end{array}$ & $0(0.0 \%)$ & $\begin{array}{l}0 \\
(0.0 \%)\end{array}$ & $\begin{array}{l}0 \\
(0.0 \%)\end{array}$ & $0(0.0 \%)$ & $\begin{array}{l}3 \\
(4.0 \%)\end{array}$ \\
\hline Unapproved tenders/ changes to tender & $0(0.0 \%)$ & $\begin{array}{l}1 \\
(50.0 \%)\end{array}$ & $\begin{array}{l}1 \\
(50.0 \%)\end{array}$ & $\begin{array}{l}0 \\
(0.0 \%)\end{array}$ & $0(0.0 \%)$ & $\begin{array}{l}2 \\
(2.7 \%)\end{array}$ \\
\hline Need to motivate for drugs & $0(0.0 \%)$ & $\begin{array}{l}1 \\
(100.0 \%)\end{array}$ & $\begin{array}{l}0 \\
(0.0 \%)\end{array}$ & $\begin{array}{l}0 \\
(0.0 \%)\end{array}$ & $0(0.0 \%)$ & $\begin{array}{l}1 \\
(1.3 \%)\end{array}$ \\
\hline $\begin{array}{l}\text { Requested quantities too small for depot to } \\
\text { supply }\end{array}$ & $0(0.0 \%)$ & $0(0.0 \%)$ & $\begin{array}{l}0 \\
(0.0 \%)\end{array}$ & $\begin{array}{l}0 \\
(0.0 \%)\end{array}$ & $1(100.0 \%)$ & $\begin{array}{l}1 \\
(1.3 \%) \\
\end{array}$ \\
\hline
\end{tabular}

\begin{tabular}{|c|c|c|c|c|c|c|}
\hline Impact of antimicrobial shortages ${ }^{\mathrm{a}}$ & $\begin{array}{l}\text { District } \\
(n=37)\end{array}$ & $\begin{array}{l}\text { Regional } \\
(n=16)\end{array}$ & $\begin{array}{l}\text { Tertiary } \\
(n=11)\end{array}$ & $\begin{array}{l}\text { Central } \\
(n=3)\end{array}$ & $\begin{array}{l}\text { Specialised } \\
(n=10)\end{array}$ & $\begin{array}{l}\text { Total } \\
(\%) \\
(n=77)\end{array}$ \\
\hline $\begin{array}{l}\text { Dispensed second/third/fourth generation } \\
\text { antimicrobials }\end{array}$ & $\begin{array}{l}35 \\
(53.8 \%)\end{array}$ & $\begin{array}{l}14 \\
(21.5 \%)\end{array}$ & $\begin{array}{l}10 \\
(15.4 \%)\end{array}$ & $\begin{array}{l}2 \\
(3.1 \%)\end{array}$ & $6(9.2 \%)$ & $\begin{array}{l}65 \\
(84.4 \%)\end{array}$ \\
\hline Dispensed more expensive alternatives & $\begin{array}{l}26 \\
(52.0 \%)\end{array}$ & $\begin{array}{l}12 \\
(24.0 \%)\end{array}$ & $\begin{array}{l}9 \\
(18.0 \%)\end{array}$ & 1 & $2(4.0 \%)$ & $\begin{array}{l}50 \\
(64.9 \%)\end{array}$ \\
\hline Turned patient away with no medication & $\begin{array}{l}14 \\
(63.6 \%)\end{array}$ & $\begin{array}{l}3 \\
(13.6 \%)\end{array}$ & $\begin{array}{l}2 \\
(9.1 \%)\end{array}$ & $\begin{array}{l}1 \\
(4.5 \%)\end{array}$ & $2(9.1 \%)$ & $\begin{array}{l}22 \\
(28.6 \%)\end{array}$ \\
\hline Referred patient to a private institution & $\begin{array}{l}10 \\
(55.6 \%)\end{array}$ & $\begin{array}{l}3 \\
(16.7 \%)\end{array}$ & $\begin{array}{l}4 \\
(22.2 \%) \\
\end{array}$ & $\begin{array}{l}0 \\
(0.0 \%)\end{array}$ & $1(5.6 \%)$ & $\begin{array}{l}18 \\
(23.4 \%) \\
\end{array}$ \\
\hline
\end{tabular}

Hospital level of care

Impact of antimicrobial shortages ${ }^{\mathrm{a}}$

$\begin{array}{llllll}\text { Procedure for reporting shortages }^{\mathrm{a}} \quad \text { District } & \text { Regional } & \text { Tertiary } & \text { Central } & \text { Specialised } & \text { Total } \\ (\%)\end{array}$

$(\mathrm{n}=35) \quad(\mathrm{n}=17) \quad(\mathrm{n}=8) \quad(\mathrm{n}=1) \quad(\mathrm{n}=7)$

\begin{tabular}{|c|c|c|c|c|c|c|}
\hline $\begin{array}{l}\text { Weekly reports to central/ provincial office or } \\
\text { district pharmacist } \\
\text { Report to PTC }\end{array}$ & $\begin{array}{l}14 \\
(53.8 \%) \\
6 \\
(40.0 \%)\end{array}$ & $\begin{array}{l}5 \\
(19.2 \%) \\
4 \\
(26.7 \%)\end{array}$ & $\begin{array}{l}3 \\
(11.5 \%) \\
3 \\
(20.0 \%)\end{array}$ & $\begin{array}{l}0 \\
(0.0 \%) \\
0 \\
(0.0 \%)\end{array}$ & $4(15.4 \%)$ & $\begin{array}{l}26 \\
(38.2 \%) \\
15 \\
(22.1 \%)\end{array}$ \\
\hline $\begin{array}{l}\text { Update on inventory management system/ out o } \\
\text { stock book }\end{array}$ & $\begin{array}{l}8 \\
(61.5 \%)\end{array}$ & $\begin{array}{l}4 \\
(30.8 \%)\end{array}$ & $\begin{array}{l}1 \\
(7.7 \%)\end{array}$ & $\begin{array}{l}0 \\
(0.0 \%)\end{array}$ & $\%)$ & $\begin{array}{l}13 \\
(19.1 \%)\end{array}$ \\
\hline $\begin{array}{l}\text { Notify prescribers via telephone, email, } \\
\text { meetings, SOPs or notices }\end{array}$ & $\begin{array}{l}8 \\
(61.5 \%)\end{array}$ & $\begin{array}{l}2 \\
(15.4 \%)\end{array}$ & $\begin{array}{l}2 \\
(15.4 \%)\end{array}$ & $\begin{array}{l}0 \\
(0.0 \%)\end{array}$ & $1(7.7 \%)$ & $\begin{array}{l}13 \\
(19.1 \%)\end{array}$ \\
\hline
\end{tabular}




\begin{tabular}{|c|c|c|c|c|c|c|}
\hline Notify pharmacy management & $\begin{array}{l}4 \\
(33.3 \%)\end{array}$ & $\begin{array}{l}4 \\
(33.3 \%)\end{array}$ & $\begin{array}{l}3 \\
(25.0 \%)\end{array}$ & $\begin{array}{l}1 \\
(8.3 \%)\end{array}$ & $0(0.0 \%)$ & $\begin{array}{l}12 \\
(17.6 \%)\end{array}$ \\
\hline Notify the depot & $\begin{array}{l}5 \\
(62.5 \%)\end{array}$ & $\begin{array}{l}2 \\
(25.0 \%)\end{array}$ & $\begin{array}{l}1 \\
(12.5 \%)\end{array}$ & $\begin{array}{l}0 \\
(0.0 \%)\end{array}$ & $0(0.0 \%)$ & $\begin{array}{l}8 \\
(11.8 \%)\end{array}$ \\
\hline Report to CEO and PTC & $\begin{array}{l}3 \\
(42.9 \%)\end{array}$ & $\begin{array}{l}1 \\
(14.3 \%)\end{array}$ & $\begin{array}{l}1 \\
(14.3 \%)\end{array}$ & $\begin{array}{l}0 \\
(0.0 \%)\end{array}$ & $2(28.6 \%)$ & $\begin{array}{l}7 \\
(10.3 \%)\end{array}$ \\
\hline Not available & $\begin{array}{l}3 \\
(100.0 \%)\end{array}$ & $0(0.0 \%)$ & $\begin{array}{l}0 \\
(0.0 \%)\end{array}$ & $\begin{array}{l}0 \\
(0.0 \%)\end{array}$ & $0(0.0 \%)$ & $\begin{array}{l}3 \\
(4.4 \%)\end{array}$ \\
\hline
\end{tabular}

${ }^{\mathrm{a}}$ More than one response option provided

CEO: Chief Executive Officer; PTC: Pharmacy and Therapeutics Committee; SOP: Standard Operating Procedure

A considerable number of facilities had active PTCs (86.9\%) and Antimicrobial Stewardship Committees (AMSCs) (70.2\%). Most respondents were members of PTCs (77.4\%) and AMSCs (51.2\%).

\section{Therapeutic interchange policies}

Less than half (42.4\%) of respondents stated they had therapeutic interchange policies in place in their hospitals, of whom 30.2\% reported that these were documented (Table 5). Pre-consultation with the prescriber before substitution was a requirement in $95.2 \%$ of the facilities. The extent of policies, their development, actions taken by pharmacist, and substitution procedures followed in the absence of policies are documented in Table 5.

Table 5 Therapeutic interchange process by hospital level of care 


\begin{tabular}{|c|c|c|c|c|c|c|}
\hline & Hospital l & vel of care & & & & \\
\hline Therapeutic interchange policy description & $\begin{array}{l}\text { District } \\
(\mathrm{n}=18)\end{array}$ & $\begin{array}{l}\text { Regional } \\
(\mathrm{n}=11)\end{array}$ & $\begin{array}{l}\text { Tertiary } \\
(\mathrm{n}=5)\end{array}$ & $\begin{array}{l}\text { Central } \\
(\mathrm{n}=1)\end{array}$ & $\begin{array}{l}\text { Specialised } \\
(\mathrm{n}=4)\end{array}$ & $\begin{array}{l}\text { Total } \\
(\%) \\
(n=39)\end{array}$ \\
\hline Memo from National Department of Health & $\begin{array}{l}6 \\
(46.2 \%)\end{array}$ & $\begin{array}{l}5 \\
(38.5 \%)\end{array}$ & $\begin{array}{l}1 \\
(7.7 \%)\end{array}$ & $1(7.7 \%)$ & $0(0.0 \%)$ & $\begin{array}{l}13 \\
(33.3 \%)\end{array}$ \\
\hline SOP/guidelines from PTCs & $\begin{array}{l}7 \\
(77.8 \%)\end{array}$ & $\begin{array}{l}2 \\
(22.2 \%)\end{array}$ & $\begin{array}{l}0 \\
(0.0 \%)\end{array}$ & $0(0.0 \%)$ & $0(0.0 \%)$ & $\begin{array}{l}9 \\
(23.1 \%)\end{array}$ \\
\hline Not available & $\begin{array}{l}1 \\
(14.3 \%)\end{array}$ & $\begin{array}{l}1 \\
(14.3 \%)\end{array}$ & $3(42.9$ & $0(0.0 \%)$ & $2(28.6 \%)$ & $\begin{array}{l}7 \\
(17.9 \%)\end{array}$ \\
\hline $\begin{array}{l}\text { Hospital notice with } \\
\text { alternatives/supplementary list }\end{array}$ & $\begin{array}{l}2 \\
(50.0 \%)\end{array}$ & $\begin{array}{l}2 \\
(50.0 \%)\end{array}$ & $\begin{array}{l}0 \\
(0.0 \%)\end{array}$ & $0(0.0 \%)$ & $0(0.0 \%)$ & $\begin{array}{l}4 \\
(10.3 \%)\end{array}$ \\
\hline $\begin{array}{l}\text { Internal arrangement between pharmacists } \\
\text { and prescribers }\end{array}$ & $\begin{array}{l}1 \\
(25.0 \%)\end{array}$ & $\begin{array}{l}1 \\
(25.0 \%)\end{array}$ & $\begin{array}{l}0 \\
(0.0 \%)\end{array}$ & $0(0.0 \%)$ & $2(50.0 \%)$ & $\begin{array}{l}4 \\
(10.3 \%)\end{array}$ \\
\hline Alternatives from the depot & $\begin{array}{l}1 \\
(50.0 \%) \\
\end{array}$ & $0(0.0 \%)$ & $\begin{array}{l}1 \\
(50.0 \%) \\
\end{array}$ & $0(0.0 \%)$ & $0(0.0 \%)$ & $\begin{array}{l}2 \\
(5.1 \%) \\
\end{array}$ \\
\hline $\begin{array}{l}\text { Health personnel responsible for development } \\
\text { of therapeutic interchange policies }{ }^{a}\end{array}$ & $\begin{array}{l}\text { District } \\
(\mathrm{n}=39)\end{array}$ & $\begin{array}{l}\text { Regional } \\
(n=17)\end{array}$ & $\begin{array}{l}\text { Tertiary } \\
(n=11)\end{array}$ & $\begin{array}{l}\text { Provincial } \\
(n=3)\end{array}$ & $\begin{array}{l}\text { Specialised } \\
(n=10)\end{array}$ & $\begin{array}{l}\text { Total } \\
(\%) \\
(\mathrm{n}=82) \\
\end{array}$ \\
\hline Pharmacy and Therapeutics Committee & $\begin{array}{l}25 \\
(61.0 \%)\end{array}$ & $\begin{array}{l}6 \\
(14.6 \%)\end{array}$ & $\begin{array}{l}3 \\
(7.3 \%)\end{array}$ & $3(7.3 \%)$ & $4(9.8 \%)$ & $\begin{array}{l}41 \\
(50.0 \%)\end{array}$ \\
\hline Pharmacist & $\begin{array}{l}12 \\
(46.2 \%)\end{array}$ & $\begin{array}{l}5 \\
(19.2 \%)\end{array}$ & $\begin{array}{l}5 \\
(19.2 \%)\end{array}$ & $2(7.7 \%)$ & $2(7.7 \%)$ & $\begin{array}{l}26 \\
(31.7 \%)\end{array}$ \\
\hline Prescribers & $\begin{array}{l}9 \\
(45.0 \%)\end{array}$ & $\begin{array}{l}3 \\
(15.0 \%)\end{array}$ & $\begin{array}{l}3 \\
(15.0 \%)\end{array}$ & $1(5.0 \%)$ & $4(20.0 \%)$ & $\begin{array}{l}20 \\
(24.4 \%)\end{array}$ \\
\hline National Department of Health & $\begin{array}{l}1 \\
(33.3 \%)\end{array}$ & $\begin{array}{l}2 \\
(66.7 \%)\end{array}$ & $\begin{array}{l}0 \\
(0.0 \%)\end{array}$ & $0(0.0 \%)$ & $0(0.0 \%)$ & $\begin{array}{l}3 \\
(3.7 \%)\end{array}$ \\
\hline Microbiologist & $0(0.0 \%)$ & $\begin{array}{l}2 \\
(100.0 \%)\end{array}$ & $\begin{array}{l}0 \\
(0.0 \%)\end{array}$ & $0(0.0 \%)$ & $0(0.0 \%)$ & $\begin{array}{l}2 \\
(2.4 \%)\end{array}$ \\
\hline Operational manager (registered nurses) & $\begin{array}{l}1 \\
(100.0 \%)\end{array}$ & $0(0.0 \%)$ & $\begin{array}{l}0 \\
(0.0 \%)\end{array}$ & $0(0.0 \%)$ & $0(0.0 \%)$ & $\begin{array}{l}1 \\
(1.2 \%)\end{array}$ \\
\hline Not applicable & $\begin{array}{l}10 \\
(45.5 \%) \\
\end{array}$ & $\begin{array}{l}6 \\
(27.3 \%) \\
\end{array}$ & $\begin{array}{l}4 \\
(18.2 \%) \\
\end{array}$ & $0(0.0 \%)$ & $2(9.1 \%)$ & $\begin{array}{l}22 \\
(26.8 \%) \\
\end{array}$ \\
\hline $\begin{array}{l}\text { Actions taken by the pharmacist during the } \\
\text { therapeutic interchange process }{ }^{\mathrm{a}}\end{array}$ & $\begin{array}{l}\text { District } \\
(\mathrm{n}=41)\end{array}$ & $\begin{array}{l}\text { Regional } \\
(n=17)\end{array}$ & $\begin{array}{l}\text { Tertiary } \\
(\mathrm{n}=11)\end{array}$ & $\begin{array}{l}\text { Provincial } \\
(n=3)\end{array}$ & $\begin{array}{l}\text { Specialised } \\
(n=10)\end{array}$ & $\begin{array}{l}\begin{array}{l}\text { Total } \\
(\%)\end{array} \\
(\mathrm{n}=82) \\
\end{array}$ \\
\hline $\begin{array}{l}\text { Communicate with other health professionals } \\
\text { regarding any ongoing medicine shortages } \\
\text { and available substitutions }\end{array}$ & $\begin{array}{l}39 \\
(50.6 \%)\end{array}$ & $\begin{array}{l}16 \\
(20.8 \%)\end{array}$ & $\begin{array}{l}10 \\
(13.0 \%)\end{array}$ & $2(2.6 \%)$ & $10(13.0 \%)$ & $\begin{array}{l}77 \\
(93.9 \%)\end{array}$ \\
\hline Keep a record of the interchange & $\begin{array}{l}25 \\
(49.0 \%)\end{array}$ & $\begin{array}{l}10 \\
(19.6 \%)\end{array}$ & $\begin{array}{l}8 \\
(15.7 \%)\end{array}$ & $2(3.9 \%)$ & $6(11.8 \%)$ & $\begin{array}{l}51 \\
(62.2 \%)\end{array}$ \\
\hline Patient counselling & $\begin{array}{l}28 \\
(68.3 \%)\end{array}$ & $4(9.8 \%)$ & $\begin{array}{l}6 \\
(14.6 \%)\end{array}$ & $1(2.4 \%)$ & $2(4.8 \%)$ & $\begin{array}{l}41 \\
(50.0 \%)\end{array}$ \\
\hline None & $\begin{array}{l}2 \\
(100.0 \%)\end{array}$ & $0(0.0 \%)$ & $\begin{array}{l}0 \\
(0.0 \%)\end{array}$ & $0(0.0 \%)$ & $0(0.0 \%)$ & $\begin{array}{l}2 \\
(2.4 \%)\end{array}$ \\
\hline $\begin{array}{l}\text { Provide prescribers with information on new } \\
\text { therapeutic equivalent and correct dosages }\end{array}$ & $\begin{array}{l}1 \\
(100.0 \%)\end{array}$ & $0(0.0 \%)$ & $\begin{array}{l}0 \\
(0.0 \%)\end{array}$ & $0(0.0 \%)$ & $0(0.0 \%)$ & $\begin{array}{l}1 \\
(1.2 \%)\end{array}$ \\
\hline $\begin{array}{l}\text { Antimicrobial substitution procedures, } \\
\text { followed by pharmacists, in the absence of } \\
\text { therapeutic interchange policies }^{\mathrm{a}}\end{array}$ & $\begin{array}{l}\text { District } \\
(\mathrm{n}=42)\end{array}$ & $\begin{array}{l}\text { Regional } \\
(n=17)\end{array}$ & $\begin{array}{l}\text { Tertiary } \\
(\mathrm{n}=11)\end{array}$ & $\begin{array}{l}\text { Central } \\
(\mathrm{n}=2)\end{array}$ & $\begin{array}{l}\text { Specialised } \\
(\mathrm{n}=9)\end{array}$ & $\begin{array}{l}\text { Total } \\
(\%)\end{array}$ \\
\hline
\end{tabular}




\begin{tabular}{|c|c|c|c|c|c|c|}
\hline $\begin{array}{l}\text { Call to notify the prescriber, avail the options } \\
\text { and they choose/ endorse change before } \\
\text { dispensing alternative }\end{array}$ & $\begin{array}{l}38 \\
(52.8 \%)\end{array}$ & $\begin{array}{l}14 \\
(19.4 \%)\end{array}$ & $\begin{array}{l}10 \\
(13.9 \%)\end{array}$ & $3(4.2 \%)$ & $7(9.7 \%)$ & $\begin{array}{l}72 \\
(88.9 \%)\end{array}$ \\
\hline $\begin{array}{l}\text { Send a written memo to the prescribers } \\
\text { supplying the available options }\end{array}$ & $\begin{array}{l}19 \\
(40.4 \%)\end{array}$ & $\begin{array}{l}14 \\
(29.8 \%)\end{array}$ & $\begin{array}{l}7 \\
(14.9 \%)\end{array}$ & $3(6.4 \%)$ & $4(8.5 \%)$ & $\begin{array}{l}47 \\
(58.0 \%)\end{array}$ \\
\hline Send the patient back to the prescriber & $\begin{array}{l}10 \\
(45.5 \%)\end{array}$ & $\begin{array}{l}7 \\
(31.8 \%)\end{array}$ & $\begin{array}{l}2 \\
(9.1 \%)\end{array}$ & $1(4.5 \%)$ & $2(9.1 \%)$ & $\begin{array}{l}22 \\
(27.2 \%)\end{array}$ \\
\hline $\begin{array}{l}\text { Consult pharmacists at tertiary level for } \\
\text { alternatives }\end{array}$ & $\begin{array}{l}1 \\
(100.0 \%)\end{array}$ & $0(0.0 \%)$ & $\begin{array}{l}0 \\
(0.0 \%)\end{array}$ & $0(0.0 \%)$ & $0(0.0 \%)$ & $\begin{array}{l}1 \\
(1.2 \%)\end{array}$ \\
\hline Borrow or buy out from other institutions & $\begin{array}{l}1 \\
(100.0 \%)\end{array}$ & $0(0.0 \%)$ & $\begin{array}{l}0 \\
(0.0 \%)\end{array}$ & $0(0.0 \%)$ & $0(0.0 \%)$ & $\begin{array}{l}1 \\
(1.2 \%)\end{array}$ \\
\hline $\begin{array}{l}\text { Urgent meeting by Rational Medicines Use } \\
\text { Committee to discuss way forward }\end{array}$ & $\begin{array}{l}1 \\
(100.0 \%)\end{array}$ & $0(0.0 \%)$ & $\begin{array}{l}0 \\
(0.0 \%)\end{array}$ & $0(0.0 \%)$ & $0(0.0 \%)$ & $\begin{array}{l}1 \\
(1.2 \%)\end{array}$ \\
\hline
\end{tabular}

$\mathrm{a}_{\text {More than one response option provided }}$

\section{Role of the pharmacist in therapeutic interchange}

More than a third of the respondents (37.1\%) said the pharmacist should communicate with prescribers regarding suitable therapeutic options when there are shortages, as well as facilitate the development of therapeutic interchange policies (22.9\%) to improve subsequent care.

Table 6 Perceived role of the pharmacist in the therapeutic interchange process

\begin{tabular}{|c|c|c|c|c|c|c|}
\hline \multirow[t]{3}{*}{ Role of the pharmacist } & \multicolumn{5}{|c|}{ Hospital level of care } & \multirow{3}{*}{$\begin{array}{l}\text { Total } \\
(\%) \\
(\mathrm{n}=70)\end{array}$} \\
\hline & District & Regional & Tertiary & Central & Specialised & \\
\hline & $(n=36)$ & $(n=16)$ & $(\mathrm{n}=9)$ & $(\mathrm{n}=1)$ & $(\mathrm{n}=8)$ & \\
\hline $\begin{array}{l}\text { Share information and communicate with } \\
\text { prescribers on rational medicine use and } \\
\text { available therapeutic options }\end{array}$ & $\begin{array}{l}12 \\
(46.2 \%)\end{array}$ & $\begin{array}{l}6 \\
(23.1 \%)\end{array}$ & $\begin{array}{l}4 \\
(15.4 \%)\end{array}$ & $\begin{array}{l}1 \\
(3.8 \%)\end{array}$ & $3(11.5 \%)$ & $\begin{array}{l}26 \\
(37.1 \%)\end{array}$ \\
\hline $\begin{array}{l}\text { Facilitate therapeutic interchange policy } \\
\text { development and/or selection of therapeutic } \\
\text { alternatives in the PTC }\end{array}$ & $\begin{array}{l}11 \\
(68.8 \%)\end{array}$ & $\begin{array}{l}2 \\
(12.5 \%)\end{array}$ & $\begin{array}{l}2 \\
(12.5 \%)\end{array}$ & $\begin{array}{l}0 \\
(0.0 \%)\end{array}$ & $1(6.3 \%)$ & $\begin{array}{l}16 \\
(22.9 \%)\end{array}$ \\
\hline $\begin{array}{l}\text { Work as part of a team with clinicians to provide } \\
\text { the best therapeutic alternatives for the patient }\end{array}$ & $\begin{array}{l}6 \\
(40.0 \%)\end{array}$ & $\begin{array}{l}4 \\
(26.7 \%)\end{array}$ & $\begin{array}{l}2 \\
(13.3 \%)\end{array}$ & $\begin{array}{l}0 \\
(0.0 \%)\end{array}$ & $3(20.0 \%)$ & $\begin{array}{l}15 \\
(21.4 \%)\end{array}$ \\
\hline $\begin{array}{l}\text { Participate in research with other health care } \\
\text { professionals to make informed medicine choices }\end{array}$ & $\begin{array}{l}2 \\
(33.3 \%)\end{array}$ & $\begin{array}{l}2 \\
(33.3 \%)\end{array}$ & $\begin{array}{l}1 \\
(16.7 \%)\end{array}$ & $\begin{array}{l}0 \\
(0.0 \%)\end{array}$ & $1(16.7 \%)$ & $\begin{array}{l}6 \\
(8.6 \%)\end{array}$ \\
\hline $\begin{array}{l}\text { Interchange without consulting the prescriber to } \\
\text { reduce patient waiting times if all the patient } \\
\text { information is available }\end{array}$ & $\begin{array}{l}2 \\
(50.0 \%)\end{array}$ & $\begin{array}{l}2 \\
(50.0 \%)\end{array}$ & $\begin{array}{l}0 \\
(0.0 \%)\end{array}$ & $\begin{array}{l}0 \\
(0.0 \%)\end{array}$ & $0(0.0 \%)$ & $\begin{array}{l}4 \\
(5.7 \%)\end{array}$ \\
\hline $\begin{array}{l}\text { Educate and counsel patients on changes to their } \\
\text { treatment }\end{array}$ & $\begin{array}{l}3 \\
(100.0 \%)\end{array}$ & $0(0.0 \%)$ & $\begin{array}{l}0 \\
(0.0 \%)\end{array}$ & $\begin{array}{l}0 \\
(0.0 \%) \\
\end{array}$ & $0(0.0 \%)$ & $\begin{array}{l}3 \\
(4.3 \%) \\
\end{array}$ \\
\hline
\end{tabular}

PTC: Pharmacy and Therapeutics Committee 


\section{Discussion}

We believe this is the first comprehensive study among public sector hospitals in South Africa to review the current situation regarding therapeutic interchange policies following NDoH guidance [25]. The response rate of $33.5 \%$ despite sending up to 12 reminders was similar to one study in the US (40\%) [31]; however, higher than studies in Europe and another study in the US with response rates of $22 \%$ and $13 \%$ respectively $[29,32]$. Pharmacy managers provided the highest number of responses (Table 2), similar to a study conducted in the US [33].

The majority of participating hospitals had experienced antimicrobial shortages in the preceding six months, similar to studies conducted in Australia, Europe and the US [32,34]. Shortages were either reported to the pharmacy manager, the PTC, hospital management or the provincial office by the pharmacists, similar to the findings in a US survey [31]. It is important that shortages are reported to the relevant authorities and immediately communicated to prescribers so that pertinent strategies can be urgently identified and implemented.

The majority of shortages reported were for penicillins followed by cephalosporins (Table 3), similar to findings in the US, Europe and Australia [10, 11, 32, 34]. Most of the shortages lasted more than 40 days. This is a concern although shortages between one week and over a year have been seen in Europe and the US $[11,32]$. Pharmaceutical companies with supply or capacity problems, an inefficient supply system, poor stock control and financial resources were the main reasons for shortages (Table 4). However, new initiatives have recently been introduced in South Africa to help improve stock control, and we will be researching their impact in future studies [17].

Most pharmacists reported they resorted to dispensing later generation antimicrobials or more expensive alternatives when faced with shortages, similar to studies in Europe and Australia [32, 34]. This though may potentially hinder AMS efforts as shortages of one antimicrobial can result in shortages of others used for substitution, potentially negatively impact on patient outcomes [35].

Encouragingly, the majority of the respondents said their hospitals had active PTCs and AMSCs. This is welcomed especially as one of the aims and objectives of the South African National Drug Policy was to establish and strengthen PTCs in all hospitals $[23,24,36]$. However, of concern was that less than half of the respondents $(42.4 \%)$ stated their hospitals had therapeutic interchange policies. Of these, only nine had policies or substitution guidelines from their institutional PTCs. This is consistent though with a recent survey conducted among public sector hospitals in South Africa which found that the development and implementation of guidelines other than the formulary is not a primary function of hospital PTCs [23]. In addition, only $33 \%$ of participants referred to the $\mathrm{NDoH}$ notice to develop their therapeutic 
interchange policies. This needs to be addressed as it is difficult to adhere to and revise guidelines that are not documented and actively communicated.

Half of the respondents stated that therapeutic interchange policies were the responsibility of PTCs, with PTCs generally considered the most ideal setting for such programmes which is encouraging as they can ensure that any guidelines are based on scientific evidence and any national Essential Medicine List [17, 37-39]. Several actions were reported as part of the pharmacist's practice during substitution (Table 5) including communication with other health professionals and providing information on available alternatives. This is also important as decisions should be made in collaboration with all key stakeholders [35]. Encouragingly, very few respondents stated they substituted without consulting the prescriber, with $92.5 \%$ stating that pre-consultation with the prescriber was a requirement before substitution in their hospital. This is important for building trust among teams. The opposite was observed in the US where $67 \%$ of institutions performed automatic substitutions preapproved by PTCs [31]. More than a quarter of respondents sent the patient back to the prescriber when a prescribed medicine was unavailable, implying that shortages were not always communicated in a timely manner. This needs to be addressed as it is important that pharmacists actively communicate with prescribers and help jointly develop therapeutic interchange policies to optimize patient care and minimize patient inconvenience $[22,40]$.

Encouragingly, keeping a record of the interchange was undertaken by more than half of the respondents. Encouraging as well was that respondents emphasised the importance of communication with prescribers, active participation in research related to substitution decisions as well as facilitation of therapeutic interchange policy development (Table 6) in going forward. We will be building on this in future studies. Finally, some pharmacists highlighted the importance of effective communication channels between the National/Provincial Department of Health and health facilities, which is consistent with the WHO's recommendation to scale up systems at a national level that collect and monitor data on medicine availability for better evidence-based policy making [41].

The study had several limitations. We acknowledge there was a relatively low response rate, more than half of the respondents had less than 10 years experience, and the responses were self-reported. It is also likely that not all shortages were accounted for due to recall bias. There also appeared to be differences in the interpretation of therapeutic interchange policies among the pharmacists taking part. Despite these limitations, we believe the study was robust providing an insight into the current status of antimicrobial shortages and interchange policies among public sector hospitals in South Africa.

\section{Conclusions And Recommendations}

From our findings, it is evident that most South African public sector hospitals experience antimicrobial shortages, particularly for penicillins and cephalosporins. These shortages may last in excess of 40 days, which can affect service delivery, AMS efforts and patient outcomes. Of concern is that the practice of therapeutic interchange is currently not that common in South Africa and not entirely understood by 
pharmacists working in public sector hospitals. Efforts should be made to train PTC members on how to develop evidence-based policies to manage medicine shortages in their institutions, with pharmacists playing a key role based on their training. There should also be increased communication with depots and ordering early considering depot lead times.

We also believe based on our findings that clear processes for the effective communication of anticipated and ongoing shortages of medicines, including antimicrobials, should be established among all public sector hospitals. These hospitals should design and implement measures to ameliorate weaknesses in the inventory control process as well as minimise disruptions in medicine supply, building on current initiatives in South Africa. These measures should prioritise antimicrobial preservation given concerns with rising AMR rates in South Africa.

Furthermore, therapeutic interchange policies should be considered for implementation at various levels of care as they take into consideration the spectrum of activity, cost and associated adverse drug reactions of different antimicrobials. This can be part of ASP programmes. We hope our findings are of interest to other low to medium income countries experiencing challenges with medicine shortages especially antimicrobials in hospitals.

\section{Abbreviations}

AMR

Antimicrobial Resistance

AMS

Antimicrobial Stewardship

AMSCs

Antimicrobial Stewardship Committees

ATC

Anatomical Therapeutic Chemical Classification

CEO

Chief Executive Officer

HIV

Human Immunodeficiency Virus

MS

Page $13 / 18$ 
Microsoft Office

$\mathrm{NDoH}$

National Department of Health

PTC

Pharmacy and Therapeutics Committee

SD

Standard Deviation

SMUREC

Sefako Makgatho University Research Ethics Committee

SOP

Standard Operating Procedures

SPSS

Statistical Package for the Social Sciences

WHO

World Health Organization

\section{Declarations}

\section{Ethics approval and consent to participate}

Ethical clearance for the study was granted by the Sefako Makgatho University Research Ethics Committee (SMUREC/P/269/2017:PG), and permission obtained from the NDoH. The electronic questionnaire included a consent statement and the clearance number; completion of the questionnaire by participants was regarded as consent.

\section{Consent for publication}

Not applicable.

\section{Availability of data and material}

The datasets during and/or analysed during the current study are available from the corresponding author on reasonable request. 


\section{Competing interests}

The authors declare that they have no competing interests.

\section{Funding}

AKC conducted the study as part of a master's degree qualification and received student tuition funding from the National Research Foundation. The study was self-funded, apart from the license for the use of SurveyMonkey ${ }^{\mathrm{TM}}$, which was funded through a National Research Foundation grant.

\section{Authors' contributions}

AKC, MM and JCM conceptualised and designed the study. AKC developed the data collection tool with contribution from MM and JCM. AKC collected and analysed the data. All authors participated in the interpretation of the data. AKC and BG wrote the first draft of the manuscript. All authors commented on the manuscript and agreed on the final version.

\section{Acknowledgements}

The authors wish to acknowledge the respective Provincial Departments of Health and hospital authorities for granting permission to conduct the study. We would like to express our gratitude to all the public sector hospital pharmacists who responded to the survey for their time and valuable responses.

\section{References}

1. Wanyiri JW, Kanyi H, Maina S et al. Infectious diarrhoea in antiretroviral therapy-naïve HIV/AIDS patients in Kenya. Transactions of the Royal Society of Tropical Medicine and Hygiene.

2013;107(10):631-8.

2. Global, regional, and national burden of tuberculosis, 1990-2016: results from the Global Burden of Diseases, Injuries, and Risk Factors 2016 Study. The Lancet Infectious diseases. 2018;18(12):1329-49.

3. Dheda K, Gumbo T, Maartens $\mathrm{G}$ et al. The epidemiology, pathogenesis, transmission, diagnosis, and management of multidrug-resistant, extensively drug-resistant, and incurable tuberculosis. The Lancet Respiratory medicine. 2017.

4. Ndihokubwayo JB, Yahaya AA, Dester AT et al. Antimicrobial resistance in the African Region: Issues, challenges and actions proposed. Afr Health Mon 2013;16: 27-30.

5. Jinks T, Lee N, Sharland $M$ et al. A time for action: antimicrobial resistance needs global response. Bull World Health Organ. 2016;94(8):558-a.

6. Cox JA, Vlieghe $E$, Mendelson $M$ et al. Antibiotic stewardship in low- and middle-income countries: the same but different? Clinical microbiology and infection. 2017;23(11):812-8. 
7. Mendelson M, Matsoso M. THE SOUTH AFRICAN ANTIMICROBIAL RESISTANCE STRATEGY FRAMEWORK. AMR CONTROL 2015:54-61.

8. Schellack N, Bronkhorst E, Coetzee R et al. SASOCP position statement on the pharmacist's role in antibiotic stewardship 2018. South African Journal of Infectious Disease 2018;33(1):28-35.

9. Valiquette L, Laupland KB. Antimicrobial shortages: Another hurdle for clinicians. The Canadian journal of infectious diseases \& medical microbiology. 2015;26(2):67-8.

10. Members of the Emerging Infections Network, Gundlapalli AV et al. Antimicrobial Agent Shortages: The New Norm for Infectious Diseases Physicians. Open Forum Inf Dis. 2018;5(4).

11. Quadri F, Mazer-Amirshahi M, Fox ER et al. Antibacterial drug shortages from 2001 to 2013: implications for clinical practice. Clinical infectious diseases. 2015;60(12):1737-42.

12. Gross AE, Gupta V, Tabak YP et al. The Effect of a Piperacillin/Tazobactam Shortage on Antimicrobial Prescribing and Clostridium difficile Risk in 88 US Medical Centers. Clinical Infectious Diseases. 2017;65(4):613-8.

13. Plüss-Suard C, Pannatier A, Ruffieux $C$ et al. Changes in the use of broad-spectrum antibiotics after cefepime shortage: a time series analysis. Antimicrobial agents and chemotherapy. 2012;56(2):989-94.

14. Hodes R, Price I, Bungane $\mathrm{N}$ et al. How front-line healthcare workers respond to stock-outs of essential medicines in the Eastern Cape Province of South Africa. S Afr Med J 2017;107(9):738-40.

15. Gonzalez LL. Medicine shortages: A hard diagnosis. 2015. Available at URL: https://www.healthe.org.za/2015/11/16/medicine-shortages-a-hard-diagnosis/.

16. Stop Stock Outs. 2015 Stock Outs National Survey - Third Annual Report - South Africa. 2016. Available at: http://www.sahivsoc.org/Files/2015_stock_outs_national_survey.pdf.

17. Meyer JC, Schellack N, Stokes J et al. Ongoing Initiatives to Improve the Quality and Efficiency of Medicine Use within the Public Healthcare System in South Africa; A Preliminary Study. Frontiers in pharmacology. 2017;8:751.

18. McKenzie A, McCann T. EVALUATION OF THE STOP STOCK OUTS PROJECT (SSP), SOUTH AFRICA. 2016.

19. Christopher E. Essential Medication Stock Outs Persist in South Africa. 2016. Available at URL: https://africahealthnews.com/essential-medication-stock-outs-persist-south-africa/.

20. Gundlapalli AV, Beekmann SE, Graham DR, Polgreen PM. Perspectives and concerns regarding antimicrobial agent shortages among infectious disease specialists. Diagnostic microbiology and infectious disease. 2013;75(3):256-9. 
21. Pulcini $C$, Beovic B, Beraud $G$ et al. Ensuring universal access to old antibiotics: a critical but neglected priority. Clinical microbiology and infection. 2017;23(9):590-2.

22. Tyler LS, Cole SW, May JR et al. ASHP guidelines on the pharmacy and therapeutics committee and the formulary system. AJHP. 2008;65(13):1272-83.

23. Mashaba TP, Matlala M, Godman B, Meyer JC. Implementation and monitoring of decisions by pharmacy and therapeutics committees in South African public sector hospitals. Expert review of clinical pharmacology. 2019;12(2):159-68.

24. Matlala M, Gous AG, Godman B, Meyer JC. Structure and activities of pharmacy and therapeutics committees among public hospitals in South Africa; findings and implications. Expert review of clinical pharmacology. 2017;10(11):1273-80.

25. Department of Health, Republic of South Africa. POLICY FOR CLASSIFYING MEDICINES INTO THERAPEUTIC CLASSES FOR PURPOSES OF THERAPEUTIC INTERCHANGE. 2017.

26. Gray T, Bertch K, Galt K et al. Guidelines for therapeutic interchange-2004. Pharmacotherapy. 2005;25(11):1666-80.

27. Kaakeh R, Sweet BV, Reilly C et al. Impact of drug shortages on U.S. health systems. AJHP. 2011;68(19):1811-9.

28. Griffith MM, Pentoney Z, Scheetz MH. Antimicrobial drug shortages: a crisis amidst the epidemic and the need for antimicrobial stewardship efforts to lessen the effects. Pharmacotherapy. 2012;32(8):665-7.

29. McLaughlin M, Kotis $\mathrm{D}$, Thomson $\mathrm{K}$ et al. Effects on patient care caused by drug shortages: a survey. JMCP. 2013;19(9):783-8.

30. WHO. WHO Collaborating Centre for Drug Statistics Methodology. Guidelines for ATC Classification and DDD Assignment. 2017.

31. Caulder CR, Mehta B, Bookstaver PB et al. Impact of Drug Shortages on Health System Pharmacies in the Southeastern United States. Hospital pharmacy. 2015;50(4):279-86.

32. Harbarth S, Filius PM, Natsch S et al. Shortage of antimicrobial agents in Europe: results of an international survey, abstr P-1105. Abstr. 17th Euro. Cong. Clin. Microbiol. Infect. Dis 2007. Munich, Germany.

33. Institute for Safe Medication Practices. Drug shortages continue to compromise patient care. 2018. Available at URL: https://www.ismp.org/resources/drug-shortages-continue-compromise-patient-care.

34. The Society of Hospital Pharmacists of Australia(SHPA). Medicine shortages in Australia: A snapshot of shortages in Australian hospitals. 2017. 
35. Ventola CL. The drug shortage crisis in the United States: causes, impact, and management strategies. P \& T. 2011;36(11):740-57.

36. NDOH. National Drug Policy for South Africa. Available at URL:

http://apps.who.int/medicinedocs/documents/s17744en/s17744en.pdf

37. Nakwatumbah S, Kibuule D, Godman B et al. Compliance to guidelines for the prescribing of antibiotics in acute infections at Namibia's national referral hospital: a pilot study and the implications. Expert review of anti-infective therapy. 2017;15(7):713-21.

38. van der Sandt N, Schellack N, Mabope LA et al. Surgical Antimicrobial Prophylaxis Among Pediatric Patients in South Africa Comparing Two Healthcare Settings. The Pediatric infectious disease journal. 2018.

39. Okoth C, Opanga S, Okalebo F et al. Point prevalence survey of antibiotic use and resistance at a referral hospital in Kenya: findings and implications. Hospital practice. 2018;46(3):128-36.

40. Alomi YA, Alghamdi SJ, Alattyh RA et al. National Survey of Pharmacy Practice at MOH Hospitals in Saudi Arabia 2016-2017: Prescribing and Medication Management. J Pharm Pract Community Med 2018;4(1s): S54-S9.

41. World Health Organization. Addressing the global shortage of, and access to, medicines and vaccines: Report by the Director-General. 2018. Available at http://apps.who.int/gb/ebwha/pdf_files/EB142/B142_13-en.pdf. 